declared, Alessandro Consolaro Grant/research support from: AbbVie, Pfizer, Angelo Ravelli Grant/research support from: Angelini, AbbVie, Bristol-Myers Squibb, Johnson \& Johnson, Novartis, Pfizer, Reckitt Benkiser, and Roche, Consultant for: Angelini, AbbVie, Bristol-Myers Squibb, Johnson \& Johnson, Novartis, Pfizer, Reckitt Benkiser, and Roche, Speakers bureau: Angelini, AbbVie, Bristol-Myers Squibb, Johnson \& Johnson, Novartis, Pfizer, Reckitt Benkiser, and Roche, Clara Malattia: None declared

DOI: 10.1136/annrheumdis-2019-eular.7082

\section{THU0595 DEVELOPMENT OF AN AUTOMATED SEGMENTATION ALGORITHM TO IDENTIFY BONES OF THE HAND}

Adwaye Rambojun ${ }^{1}$, William Tillett ${ }^{2,3}$, Neill Campbell ${ }^{4}$, Tony Shardlow ${ }^{1}$. ${ }^{1}$ University of Bath, Mathematical Sciences, Bath, United Kingdom; ${ }^{2}$ University of Bath, Pharmacology, Bath, United Kingdom; ${ }^{3}$ Royal National Hospital For Rheumatic Diseases, Bath, United Kingdom; ${ }^{4}$ University of Bath, Computer Science, Bath, United Kingdom

Background: The evaluation of structural damage with plain radiography is important to clinicians and patients. Standard scoring methods include the Sharp-van der Heijde (SVdH) and Ratingen methods [1] however these systems are time-consuming. Therefore, it is difficult to perform large cohort studies. We set out to develop an automated algorithm to identify bones on plain radiographs as a step towards developing automated quantification of structural damage for use on large datasets.

Objectives: To develop a novel algorithm to segment outlines of finger bones in hand radiographs.

Methods: 101 hand radiographs were gathered from the Bath longitudinal cohort (UK). All patients fulfilled the CASPAR criteria for Psoriatic Arthiritis (PSA). None of the patients had damage on SVdH and Ratingen scoring (blinded). The metacarpal (MC), proximal phalanx (PP), middle phalanx (MP), and distal phalanx (DP) in the right index finger were delineated by a rheumatologist. These outlines were used to build a statistical model of the shape using a Gaussian Process Latent Variable Model (GPLVM) [2]. Bones are segmented by matching the shape on a radiograph to the statistical model.

Results: The performance of the matching algorithm was compared with a traditional algorithm (snakes) using the Adjusted Rand Score (ARND). The ARND score measures the similarity of the segmentation with the ground truth. A perfect segmentation has a score close to 1 . We tested the algorithm on $9 \mathrm{PP}, 9 \mathrm{MP}$ and $8 \mathrm{DP}$ and $6 \mathrm{MC}$ bones in the right index finger. The results are reported in table 1 . We report a mean improvement in ARAND of $0.19,0.87,0.43$ and 0.30 for the PP, MP, DP and MC respectively.

Conclusion: We report a reliable algorithm for the identification of metacarpal, proximal, middle and distal phalanx bones of the hand. Future work will focus on using the output of the segmentation algorithm to track damage progression over time.

\section{REFERENCES:}

[1] Van der Heijde, D., PAULUS, H., and SHEKELLE, P. (2000). How to read radiographs according to the Sharp/vander Heijde method. Discussion: Heterogeneity in rheumatoid arthritis radiographic trials. Issues to consider in a metaanalysis. Journal of rheumatology, 27(1):261-263.

[2] Titsias, M. K. and Lawrence, N. D. (2010). Bayesian Gaussian process latent variable model. In International Conference on Artificial Intelligence and Statistics, pages 844-851.

Table 1. Adjusted RAND scores for comparing our algorithm to a traditional one (snakes)

\begin{tabular}{lccc}
\hline & Bone & $\begin{array}{c}\text { Snakes } \\
\text { ARAND }\end{array}$ & $\begin{array}{c}\text { Shape matching } \\
\text { ARAND }\end{array}$ \\
\hline Case 1 & PP & 0.70 & 0.95 \\
Case 2 & PP & 0.89 & 0.96 \\
Case 3 & PP & 0.82 & 0.96 \\
Case 4 & PP & 0.72 & 0.94 \\
Case 5 & PP & 0.53 & 0.96 \\
Case 6 & PP & 0.87 & 0.97 \\
Case 7 & PP & 0.79 & 0.96 \\
Case 8 & PP & 0.74 & 0.96 \\
Case 9 & PP & 0.88 & 0.97 \\
Case 1 & MP & 0.79 & 0.95 \\
Case 2 & MP & 0.75 & 0.95 \\
Case 3 & MP & 0.75 & 0.94
\end{tabular}

$\begin{array}{llll}\text { Case } 4 & \text { MP } & 0.72 & 0.95 \\ \text { Case 5 } & \text { MP } & 0.77 & 0.96 \\ \text { Case 6 } & \text { MP } & 0.51 & 0.96 \\ \text { Case 7 } & \text { MP } & 0.76 & 0.96 \\ \text { Case 8 } & \text { MP } & 0.77 & 0.96 \\ \text { Case 9 } & \text { MP } & 0.83 & 0.97 \\ \text { Case } & \text { MP } & 0.77 & 0.93\end{array}$

10

Key: Adjusted Rand Score (ARND) score measures the similarity of the segmentation with the ground truth. A perfect segmentation has a score close to 1. Metacarpal (MC), proximal phalanx (PP), middle phalanx (MP), and distal phalanx (DP)

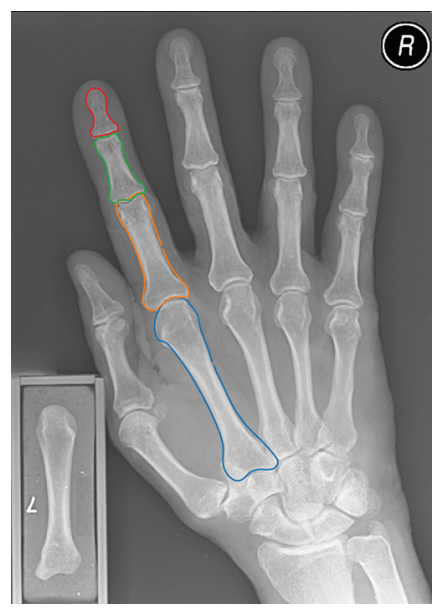

Figure 1. Shape matching algorithm output demonstrating segmented outlines of the DP, MP, PP and MC in red, green, orange, and blue respectively.

Disclosure of Interests: Adwaye Rambojun: None declared, William Tillett Grant/research support from: AbbVie, Celgene, and Lilly, Consultant for: AbbVie, Celgene, Lilly, Novartis, and Pfizer, Speakers bureau: Abbvie Celgene, Lilly, Janssen, Novartis, UCB, and Pfizer, Neill Campbell: None declared, Tony Shardlow: None declared

DOI: 10.1136/annrheumdis-2019-eular.2336

\section{THU0596 DIAGNOSTIC VALUE OF ULTRASOUND AND DUAL ENERGY COMPUTED TOMOGRAPHYTO ACHIEVE ACR- EULAR GOUT CLASSIFICATION CRITERIA IN REAL LIFE CLINICAL PRACTICE}

André Ramon ${ }^{1}$, Marie Schmitt ${ }^{1}$, Romaric $\mathrm{Ne}^{2}$, Pierre Emmanuel Berthod ${ }^{2}$, Hervé Devilliers ${ }^{3}$, Jean Francis Maillefert ${ }^{1}$, Paul Ornetti ${ }^{1} .{ }^{1}$ Dijon University Hospital, Rheumatology, Dijon, France; ${ }^{2}$ Dijon University Hospital, Radiology, Dijon, France; ${ }^{3}$ Dijon University Hospital, Internal Medicine, dijon, France

Background: 2015 ACR/EULAR gout classification criteria (1) include ultrasound with double contour (DC) sign as key ultrasound features and dual energy computed tomography (DECT) with evidence of urate deposition. The positivity of either DECT or ultrasound allows 4 points in addition to others clinical and biological criteria to classify as gout is $\geq 8 / 23$. However, in routine care, the imaging modality that should be promoted remains unclear between ultrasound or DECT.

Objectives: To validate a possible diagnostic algorithm for the clinical use of DECT and ultrasound in suspected gouty arthritis.

Methods: We conducted a single-center prospective study in the Rheumatology Department of Dijon University Hospital from july 2016 to december 2018, including all patients hospitalized for suspected gouty arthritis Each patient received joint aspiration if possible, an ultrasound assessment (DC sign and/or tophus) and DECT scanning of symptomatic joints All these examinations were performed blind of the clinical data and results of joint aspiration. The gold standard used for this study was the 2015 ACR/EULAR gout classification criteria. We have established two 